\title{
COVID-19 boosters in rich nations will delay vaccines for all
}

To the Editor - On 4 August 2021, Tedros Adhanom Ghebreyesus, Director-General of the World Health Organization, called for a global moratorium on booster doses of vaccination against COVID-19, until the end of September, with a goal of having $10 \%$ of every nation's population vaccinated. This comes on the heels of both Israel and Germany starting vaccine campaigns of a third dose, and provinces such as Quebec, Canada, giving third doses to provide an easier pathway for international travel. In the United States, on 12 August 2021, the US Food and Drug Administration authorized booster doses for certain immunocompromised people.

As more rich nations consider boosters, their local public-health communities need to wake up to the widening chasm of vaccine inequity and its devastating consequences, especially with the Delta variant ripping through populations. All of us need to look within and ask hard questions. Are we as a species willing to protect all humankind, or do we mostly care about optimizing protection for people in wealthy nations?

Any discussion of booster dose strategies requires an application of scientific as well as equity principles.

From a scientific perspective, a key question is how long the immune response to the mRNA vaccines lasts. Data from Pfizer show efficacy of $84 \%$ after 4 months for symptomatic COVID-19, with $97 \%$ protection against severe disease ${ }^{1}$. The Moderna vaccine has shown efficacy of over $90 \%$ at 6 months against symptomatic COVID-19². Although data on the vector-based immunizations are lacking, experiences from the United Kingdom show that the majority of complicated COVID19 cases exist among the unvaccinated, rather than those who received the AstraZeneca vaccine ${ }^{3}$. All approved vaccines seem to show reasonable efficacy against symptomatic and severe disease after infection with the Delta variant in datasets from the United Kingdom ${ }^{4}$ and Canada ${ }^{5}$.

As new variants emerge, breakthrough cases emerge, and a small number of these patients will develop complications, but no data exist that link boosters to enhanced real-life protection in the general population. Other than rare immunosuppressed patients, such as organ-transplant recipients ${ }^{6}$, the vast

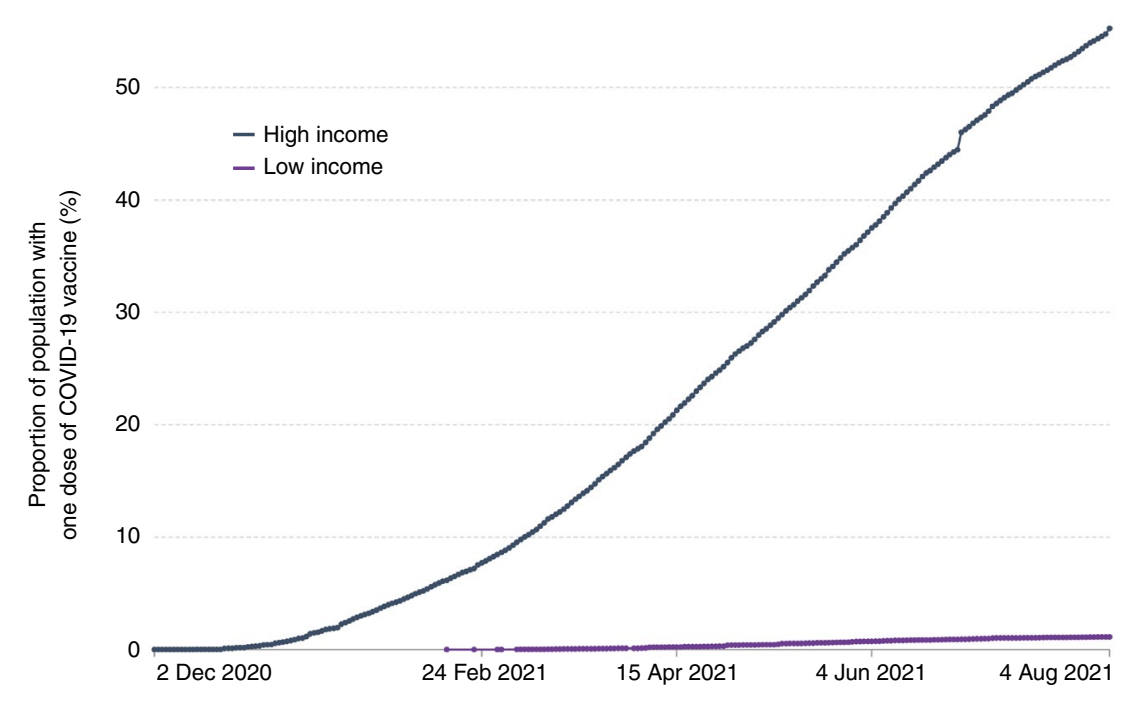

Fig. 1 | Vaccination in low- and high-income countries. Share of the population in high-income countries that has received at least one dose of COVID-19 vaccine, compared with that in low-income countries. Source: https://ourworldindata.org/.

majority of the population, including the elderly and those with medical conditions, derive adequate protection from the existing vaccine schedule. Vaccinating the unvaccinated can save many more lives than would boosting people who already have a fairly high level of protection.

From an equity perspective, there is a real risk of worsening the existing global inequity in vaccinations. There remain 3.5 billion people on this earth, nearly half of the world's population, who have not received even a first dose of vaccine. Only $2 \%$ of the African population has received even a single dose $\mathrm{e}^{7}$.

Without widespread vaccination, it is impossible to protect any nation from the catastrophic consequences of the Delta variant, a variant that is highly transmissible and is associated with severe outcomes. Although those nations afforded the luxury of vaccinations will probably deal with a relatively small amount of breakthrough infections, unvaccinated nations will deal with large-scale death, long-term disability and healthcare collapse. This was clearly demonstrated in India, where three to five million excess deaths might have occurred during the pandemic ${ }^{8}$.

India also illustrates the danger of letting the virus rip through largely unvaccinated populations: newer variants emerge quickly in such settings and can spread around the world, as the Delta variant indeed has. The United States and the United Kingdom, countries with easy access to vaccines, are now struggling with surges in infections from the Delta variant.

It is distressing that millions of healthcare workers in low-income countries are yet to be fully vaccinated. Unvaccinated healthcare workers face ongoing mortal risks in performing their duties, and thousands of healthcare workers have died already. The devastating impact of COVID-19 goes well beyond the disease itself. The pandemic is seriously disrupting routine healthcare services. For example, 23 million children missed out on basic, routine childhood vaccines in 2020 , the highest number since 2009 (ref. ${ }^{9}$ ). The death toll and suffering from this disease will be felt for years among the world's most vulnerable residents, both adults and children.

The US White House recently stated that choosing between boosters and global vaccinations is a false choice, and cited a substantial contribution to global vaccination, which has helped to alleviate the suffering. If the COVID-19 vaccine supply were unlimited, then vaccinating those who are unvaccinated in high-income 
nations and preparing for boosters to optimize protection makes sense. But currently, the vaccine supply is limited and this is a zero-sum game.

In fact, it has been a zero-sum game for some time, and will continue to be until supply is dramatically increased via the TRIPS (Trade-Related Aspects of Intellectual Property Rights) waiver and technology transfers. Since rich nations have procured a giant share of current and future doses, booster vaccination programs will mean that low-income countries will have to wait longer to get their orders fulfilled. Pharmaceutical companies will typically give priority to rich nations that are likely to pay premium prices. Booster doses in rich countries is a lucrative market.

Rich countries claimed they could both vaccinate their adolescents in early May while still contributing to global vaccine campaigns, but the reality of the situation was different. From May to August 2021, high-income countries had given a first dose of vaccine to $28 \%$ of their population, while low-income countries had given a mere $0.7 \%$ of their population their first dose (Fig. 1).

The population in high-income countries is nearly 50 times more vaccinated than that in low-income countries. Many lowand middle-income countries are awaiting contracts from manufacturers and COVAX (the vaccines pillar of the Access to COVID19 Tools (ACT) Accelerator) to be filled while dealing with a Delta wave, while other countries have more vaccine than eligible residents. In fact, several countries, including the United States, Canada, Israel and the United Kingdom, are now throwing away expired or expiring vaccines.

Given the global community in this vaccine and scientific era, it is unconscionable for the outcomes of COVID-19 to be determined simply by the country one resides in. Rich nations that have vaccinated most of their population should consider reallocation of excess vaccinations, while also being mindful of the effect of future purchases on lower-income countries awaiting orders to be fulfilled. We urge everyone, wherever they live, to become outspoken advocates for vaccine equity $^{10}$. Our collective future is at stake.

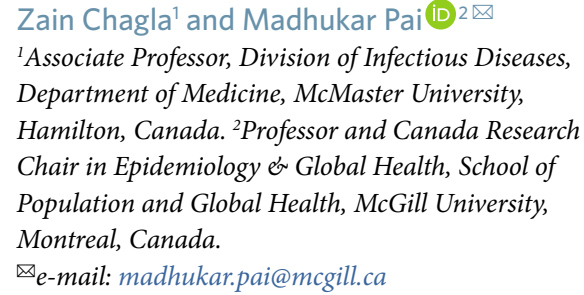

Published online: 31 August 2021

https://doi.org/10.1038/s41591-021-01494-4

References

1. Thomas, S. J. et al. Preprint at medRxiv https://doi. org/10.1101/2021.07.28.21261159 (2021)

2. Moderna. https://investors.modernatx.com/news-releases/ news-release-details/moderna-reports-second-quarter-fiscal-year2021-financial (5 August 2021).

3. Public Health England. https://assets.publishing.service.gov uk/government/uploads/system/uploads/attachment_data/ file/1005517/Technical_Briefing 19.pdf (23 July 2021).

4. Lopez Bernal, J. et al. N. Engl. J. Med. https://doi.org/10.1056/ NEJMoa2108891 (2021).

5. Nasreen, S. et al. Preprint at medRxiv https://doi. org/10.1101/2021.06.28.21259420 (2021).

6. Kamar, N. et al. N. Engl. J. Med. https://doi.org/10.1056/ NEJMc2108861 (2021).

7. Maxmen, A. Nature https://doi.org/10.1038/d41586-021-02109-1 (2021).

8. Anand, A., Sandefur, J. \& Subramanian, A. Center for Global Development https://www.cgdev.org/sites/default/files/threenew-estimates-indias-all-cause-excess-mortality-duringcovid-19-pandemic.pdf (July 2021).

9. World Health Organization. https://www.who.int/news/item/1507-2021-covid-19-pandemic-leads-to-major-backsliding-on-child hood-vaccinations-new-who-unicef-data-shows (15 July 2021).

10. Pai, M. Nature Portfolio https://naturemicrobiologycommunity. nature.com/posts/10-reasons-why-everyo ne-should-advocate-for-covid-19-vaccine-equity?channel_id=25 49-coronaviruses-past-present-and-future (15 April 2021).

\section{Author contributions}

Both authors were involved with the conceptualization, writing, and review of this Correspondence.

\section{COVID-19 vaccination gives hope to eradicate polio}

To the Editor-Before the emergence of COVID-19, polio was considered to be one of the most challenging infectious diseases of international concern, despite the consistent efforts of the Global Polio Eradication Initiative (GPEI) program to vaccinate every child in the endemic countries, Pakistan and Afghanistan. In June 2021, the GPEI launched a revised and strengthened plan 'Polio Eradication Strategy 2022-2026: Delivering on a Promise', replacing the previous plan from 2019 to 2023 , to overcome the continuing challenges in polio eradication ${ }^{1}$.

In the past five years, $67 \%$ of the total wild poliovirus type 1 (WPV1) cases were reported from Pakistan, with the remaining 33\% from Afghanistan, and all other cases worldwide were due to circulatory vaccine-derived poliovirus ${ }^{2}$. Unlike routine pediatric vaccination, polio vaccination campaigns have been particularly hampered by conspiracy theories and poor awareness about the lifesaving benefits of vaccines, at least among some parents ${ }^{3}$. Consequently, lack of vaccination and incomplete vaccination have been the main factors for the emergence of WPV1 and circulatory vaccine-derived poliovirus (cVDPV2) cases in both countries. However, the recent global vaccination drive against COVID-19 has raised public understanding of the need for vaccines in general, including polio vaccine. Although COVID-19 and polio have distinct physiological manifestations, both diseases share the commonality of vaccination as the only preventive measure.

The COVID-19 pandemic is continuously playing havoc with 211.7 million cases and 4.43 million deaths (as of 23 August 2021) ${ }^{4}$. Although the emergence of different variants of SARS-CoV-2, such as the Delta variant, has challenged control strategies, the WHO-approved vaccines have diminished the drastic situation in terms of mortality and morbidity. As of 23 August 2021, 24.6\% of the world population had been fully vaccinated, $32.7 \%$ had received at least a single dose and 33.56 million doses are being administered on daily basis ${ }^{5}$.

However, the pandemic has compromised the fragile and oversaturated healthcare systems in low- and middle-income countries, including the routine pediatric immunization against vaccine-preventable diseases. For example, inadequate vaccination against measles has listed Pakistan and Afghanistan among the top ten countries with the highest measles cases in $2021^{6}$. According to UNICEF, the temporary suspension of polio vaccination campaigning in both countries 